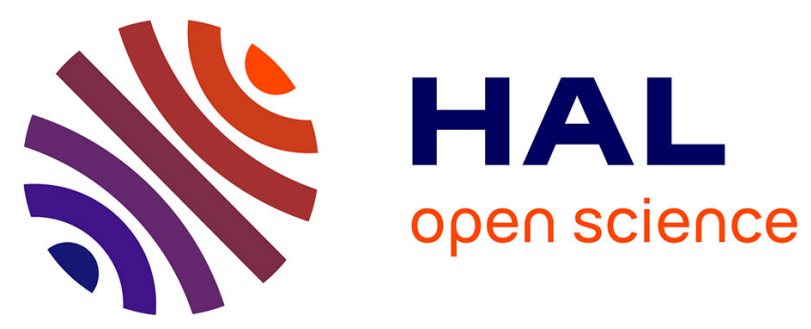

\title{
Chypre: un enjeu stratégique pour la candidature turque à l'Union européenne
}

\author{
Gilles Bertrand
}

\section{To cite this version:}

Gilles Bertrand. Chypre: un enjeu stratégique pour la candidature turque à l'Union européenne. Cahiers d'études sur la Méditerranée orientale et le monde turco-iranien, 2003, Turquie: désir d'Europe?, 36, pp.159-180. 10.3406/cemot.2003.1708 . halshs-00327863

\section{HAL Id: halshs-00327863 \\ https://shs.hal.science/halshs-00327863}

Submitted on 24 Apr 2019

HAL is a multi-disciplinary open access archive for the deposit and dissemination of scientific research documents, whether they are published or not. The documents may come from teaching and research institutions in France or abroad, or from public or private research centers.
L'archive ouverte pluridisciplinaire HAL, est destinée au dépôt et à la diffusion de documents scientifiques de niveau recherche, publiés ou non, émanant des établissements d'enseignement et de recherche français ou étrangers, des laboratoires publics ou privés.

\section{(1)(1) $\$(0)$}

Distributed under a Creative Commons Attribution - NonCommercial - ShareAlikel 4.0 


\section{Chypre : un enjeu stratégique pour la candidature turque à l'Union européenne} Gilles Bertrand

\section{Résumé}

La candidature de la Turquie à l'Union européenne a buté jusqu'en 2004 sur un obstacle de taille : l'occupation d'une partie de la république de Chypre. Ankara n'est en effet jamais parvenu à imposer sa solution (la partition) à la "communauté internationale». Le dirigeant chypriote turc Rauf Denktaş a refusé de signer le plan Annan de l'ONU, prévoyant une solution fédérale dans le cadre de l'intégration européenne. Les détenteurs du pouvoir à Ankara, civils et militaires, sont longtemps demeurés très divisés au sujet de Chypre. Mais pourquoi ? S'agissait-il d'un refus de se retirer d'une ancienne possession ottomane, la seule (avec la province d'Hatay ou Sandjak d'Alexandrette) dont l'armée turque a repris le contrôle depuis 1923 ? D'une crainte de voir le conflit reprendre, avec le risque que la communauté chypriote turque en soit, à nouveau, la principale victime ? Ou bien ce refus était-il une victoire des adversaires de l'adhésion à l'UE qui voyaient là un moyen de sauvegarder souveraineté et intérêts nationaux turcs ? Le gouvernement de R.T. Erdoğan a bouleversé la politique chypriote de la Turquie de manière à ce que ce dossier ne soit plus un obstacle à l'ouverture des négociations d'adhésion à l'UE, et ce malgré l'échec du plan Annan dû au «non» des Chypriotes grecs le 24 avril 2004.

\section{Citer ce document / Cite this document :}

Bertrand Gilles. Chypre : un enjeu stratégique pour la candidature turque à l'Union européenne. In: CEMOTI, n³6, 2003. Turquie : désir d'Europe ? pp. 159-180;

doi : https://doi.org/10.3406/cemot.2003.1708

https://www.persee.fr/doc/cemot_0764-9878_2003_num_36_1_1708

Fichier pdf généré le 19/03/2019 


\section{CHYPRE : UN ENJEU STRATEGIQUE POUR LA CANDIDATURE TURQUE A L'UNION EUROPEENNE}

Gilles BERTRAND

Résumé : La candidature de la Turquie à l'Union européenne a buté jusqu'en 2004 sur un obstacle de taille : l'occupation d'une partie de la république de Chypre. Ankara n'est en effet jamais parvenu à imposer sa solution (la partition) à la " communauté internationale ». Le dirigeant chypriote turc Rauf Denktaş a refusé de signer le plan Annan de l'ONU, prévoyant une solution fédérale dans le cadre de l'intégration européenne. Les détenteurs du pouvoir à Ankara, civils et militaires, sont longtemps demeurés très divisés au sujet de Chypre. Mais pourquoi? S'agissaitil d'un refus de se retirer d'une ancienne possession ottomane, la seule (avec la province d'Hatay ou Sandjak d'Alexandrette) dont l'armée turque a repris le contrôle depuis 1923? D'une crainte de voir le conflit reprendre, avec le risque que la communauté chypriote turque en soit, à nouveau, la principale victime? Ou bien ce refus était-il une victoire des adversaires de l'adhésion à l'UE qui voyaient là un moyen de sauvegarder souveraineté et intérêts nationaux turcs? Le gouvernement de R.T. Erdoğan a bouleversé la politique chypriote de la Turquie de manière à ce que ce dossier ne soit plus un obstacle à l'ouverture des négociations d'adhésion à l'UE, et ce malgré l'échec du plan Annan dû au «non » des Chypriotes grecs le 24 avril 2004.

Après trente ans de quasi-immobilité, la situation à Chypre a été bouleversée par une série d'événements à plusieurs niveaux : turc (dans les urnes), avec la victoire du Parti de la justice et du développement et son accession au gouvernement (3 novembre 2002); onusien, avec la présentation du plan Annan (11 novembre 2002); chypriote turc (dans la rue), avec les manifestations spectaculaires contre le régime en place et pour l'intégration européenne et la réunification (la première le 27 novembre 2002); européen, 
avec la signature du traité d'élargissement de l'Union à dix États dont la république de Chypre (16 avril 2003); chypriote, avec la décision (prise vraisemblablement à Ankara) d'ouvrir la ligne verte de démarcation aux passages des citoyens ordinaires entre les deux zones pour de courts séjours (23 avril 2003). Cependant, un an plus tard (24 avril 2004), les Chypriotes grecs ont rejeté par référendum la cinquième version du plan Annan tandis que les Chypriotes turcs l'ont approuvé. Le $1^{\text {er }}$ mai 2004, la république de Chypre entre donc dans l'Union européenne divisée et, en pratique, réduite aux seuls Chypriotes grecs.

La victoire du Parti de la justice et du développement $\left(A K P^{1}\right)$, islamiste, de Recep Tayyip Erdoğan, lors des élections législatives turques du 3 novembre 2002, est donc le premier de ces changements majeurs, et pas seulement dans la chronologie. Non seulement les partis au pouvoir durant les années 1990 ont été balayés, mais la politique vis-à-vis de Chypre a également changé, et de manière spectaculaire: ouverture de la ligne verte en avril 2003, négociations sur le plan Annan et, finalement, appel aux Chypriotes turcs à voter " oui » au référendum sur ce plan. Nous tenterons d'expliquer ici le lien entre émergence de ces nouvelles élites, marginalisations des anciennes et changement affiché quant à Chypre. Cette nouvelle politique s'appuie sur la contestation croissante des Chypriotes turcs vis-à-vis de la présence militaire turque et de l'immobilisme de leur dirigeant politique, Rauf Denktaş, le président de la "République turque de Chypre du Nord » $\left(\mathrm{KKTC}^{2}\right)$, non reconnue internationalement. Provoquée par une crise politico-financière particulièrement grave à l'été 2000, cette contestation connaît une nouvelle phase aiguë depuis la réponse négative de $R$. Denktaş au plan Annan dans les semaines suivant sa présentation (automne 2002). Les élections législatives en KKTC en décembre 2003 ont porté au pouvoir un partisan du plan Annan, le dirigeant du parti de gauche CTP ${ }^{3}$, Mehmet Ali Talât. Celui-ci a toutefois dû formé une coalition avec le DP (Demokrat Parti - Parti démocrate) dirigé par Serdar Denktaş (fils de Rauf Denktaş) qui est devenu vice-premier ministre. Mais M.A. Talât est parvenu à son objectif: le plan Annan (renégocié) a été soumis aux Chypriotes turcs qui l'ont approuvé.

Les plus grandes surprises sont donc venues d'Ankara et de Chypre-Nord. Car les décisions des organisations internationales étaient plus ou moins attendues. Le Secrétaire général des Nations unies, Kofi Annan, s'était en effet beaucoup investi dans les négociations dites intercommunautaires sur

\footnotetext{
${ }^{1}$ Adalet ve Kalkınma Partisi créé en 2001.

${ }^{2}$ Kuzey Kıbrıs Türk Cumhuriyeti, proclamée en novembre 1983.

${ }^{3}$ Cumhuriyetçi Türk Partisi - Parti républicain turc.
} 
Chypre depuis 1999. Le cadre et le rythme de ces négociations ont été dominés par la candidature de la république de Chypre à l'UE, candidature acceptée en mars 1995 mais dont le "compte à rebours" commença en mars 1998 avec l'ouverture officielle du processus d'adhésion. Le plan Annan, après "l'Ensemble d'idées » de Boutros Boutros-Ghali et les nombreux projets de résolution du conflit élaborés par les Nations unies, devait donc voir le jour avant l'adhésion à l'UE, ou jamais. La Commission et le Conseil européens, de leur côté, cachaient de moins en moins leur intention d'intégrer Chypre même divisée, notamment par la négociation d'un traité d'adhésion globale pour les dix nouveaux membres, et non un par candidat comme dans le passé. Chypre est donc embarqué sur le paquebot "élargissement", malgré le refus du plan Annan exprimé dans les urnes par les Chypriotes grecs. Il faudra donc revenir brièvement sur le plan Annan et l'intégration européenne de Chypre.

\section{L'évolution de la situation à Chypre depuis le début des années 1990, un bref rappel ${ }^{4}$}

Après l'immobilité des années 1980 (négociations sans fin et sans résultat Kyprianou-Denktaş), la décennie 1990 s'ouvre sur l'espoir suscité par « l'Ensemble d'idées » du Secrétaire général des Nations unies, Boutros Boutros-Ghali. Mais les négociations échouent une fois encore, la responsabilité en incombant clairement à $R$. Denktaş. C'est pourtant le Président de la république de Chypre depuis 1988, George Vassiliou, qui en paie le prix : il est défait par Glafcos Clerides lors de la présidentielle de 1993. Ce dernier n'accepte pas l'ouverture d'un nouveau cycle de négociations sous l'égide des Nations unies, mais choisit la voie de l'unilatéralisme pour bousculer le statu quo. C'est ainsi qu'il pose la candidature de Chypre à l'adhésion à l'UE (1993) et s'engage dans une politique de dissuasion militaire qui aboutit à une crise majeure lors de l'achat de missiles sol-air russes S-300 (1997).

Ces missiles de défense anti-aérienne, concurrents des Patriot américains, sont censés dissuader l'armée turque de lancer une offensive aérienne contre la zone sud de Chypre. L'annonce de leur achat provoque la colère des généraux turcs et de sévères mises en garde du gouvernement turc. La

\footnotetext{
${ }^{4}$ Voir Pierre Blanc, La Déchirure chypriote. Géopolitique d'une île divisée, Paris, L'Harmattan, « Histoire et Perspectives méditerranéennes », 2000, et Jean-François Drevet, Chypre en Europe, (même éditeur, même collection), 2000, ainsi que notre Étude du CERI : Gilles Bertrand, "Vingt-cinq ans après, où en est la partition de Chypre?", n' 59, octobre 1999.
} 
menace de frappes préventives contre les rampes de lancement des missiles est à peine voilée. Les Chypriotes turcs, qui ne craignent théoriquement rien de tels missiles, perçoivent très mal cette décision, y compris parmi les partisans de la réunification. L'annonce de cet achat d'armements est le point culminant d'une escalade diplomatico-militaire entre la Grèce et la république de Chypre d'une part, la Turquie d'autre part. Les gouvernements grec et de la république de Chypre ont tenté, en effet, de modifier l'équilibre des forces à Chypre par leur nouvelle alliance militaire baptisée « Doctrine de défense unique $»^{5}$. La Doctrine a été lancée officiellement en décembre 1993 par une déclaration commune du Premier ministre grec, Andréas Papandréou, et de Glafcos Clerides. Elle a pour objectif de mieux coordonner la défense de "l'espace hellénique » englobant la Grèce et la zone sud de Chypre contre «l'expansionnisme turc». Elle se traduit par des manœuvres militaires conjointes (baptisées Nikiphoros) à Chypre même, tous les mois d'octobre depuis 1994, qui répondent aux manœuvres turques (Toros). Une base aérienne a été construite à l'est de Paphos ${ }^{6}$. Les forces aériennes turques disposent, quant à elles, de la base de Geçitkale (Lefkoniko), située près de Famagouste. Mais la nouvelle base grecque n'a de sens que si elle est défendue contre les attaques aériennes, d'où l'achat des S-300 russes, après le refus américain de vendre des Patriot. Les réactions négatives de Washington et de l'UE ainsi que le nouveau cours de la diplomatie grecque sous le gouvernement de Costas Simitis conduisent finalement à la décision d'installer les S-300 en Crète plutôt qu'à Chypre (décembre 1998) ${ }^{7}$.

Il n'empêche : les relations entre les deux zones de Chypre sont alors au plus bas et la situation présente de nombreuses similarités avec celle qui règne lors des référendums du 24 avril 2004. La gauche chypriote turque critique une décision chypriote grecque de relance de la course aux armements, l'absence de consultations et d'explications, notamment entre partis proches, tels que le CTP et le parti communiste chypriote grec $(\mathrm{AKEL})^{8}$, favorable aux S-300 par suivisme des sondages ${ }^{9} \ldots$ Cette crise de

\footnotetext{
${ }^{5}$ EAD : Eniaio Amyntiko Dogma.

${ }^{6}$ Baptisée Andréas Papandréou, elle a été inaugurée le 24 janvier 1998 et elle coïncide exactement avec l'aéroport de Paphos dont les dimensions sont modestes. Elle doit accueillir des avions de chasse grecs (la république de Chypre ne dispose pas de forces aériennes) pouvant défendre l'île en cas d'attaque turque.

${ }^{7}$ Ils doivent désormais protéger le décollage des chasseurs grecs destinés à appuyer la Garde nationale chypriote en cas de reprise des hostilités avec l'armée turque, et non plus leur atterrissage à Chypre.

${ }^{8}$ Anorthotikon Komma tou Ergazomenou Laou.
} 
confiance entre partis de gauche est toujours perceptible au début de la décennie 2000 et paraît difficile à surmonter après le soutien d'AKEL au " non » au plan Annan. AKEL semble une fois encore s'être détourné de son objectif officiel de réconciliation au profit de considérations politiciennes internes à la communauté chypriote grecque. Cette rupture entre partis de gauche des deux communautés profite aux droites nationalistes, chypriote turque (qui y voit une confirmation de ses thèses sur "l'expansionnisme grec " et l'impossibilité de la réconciliation), et chypriote grecque (qui ne voit ainsi jamais remettre en question sa lourde responsabilité dans le conflit intercommunautaire de 1957 à 1974).

Cette crise des missiles S-300 surpasse celle d'août 1996, lorsque deux Chypriotes grecs avaient été tués sur la ligne verte à la suite d'une manifestation contre l'occupation. En effet, les meurtriers, ainsi que ceux des deux autres Chypriotes grecs tués cette année-là sur la ligne, sont presque immédiatement identifiés comme des citoyens turcs résidant à ChypreNord : un ministre de l'Agriculture, un policier, un soldat et des militants d'extrême-droite (« Loups gris »). Les affrontements d'août 1996 n'ont donc rien d'intercommunautaires, contrairement à ce que prétendent la presse internationale et... $\mathrm{l}^{\prime} \mathrm{ONU} \mathrm{U}^{10}$.

Surtout, ces deux crises masquent en partie l'émergence et le développement d'un mouvement bicommunautaire dynamique sur lequel nous avons déjà écrit ${ }^{11}$. Or, ce mouvement en tant que tel a avancé des idées pour une réconciliation à Chypre qui ont été reprises par l'ONU pour le plan Annan - ou qui l'ont inspiré. Ce mouvement a également préparé ce qui allait être un choc pour la majorité des Chypriotes le 23 avril 2003 : la possibilité de passer la ligne verte pour visiter l'autre zone et rencontrer les membres de l'autre communauté. L'absence de violences entre Chypriotes grecs et turcs lors de ces visites n'a ainsi rien d'étonnant : les rencontres bicommunautaires avaient depuis plus d'une décennie prouvé qu'ils pouvaient dialoguer.

Enfin, le mouvement a contribué, côté chypriote turc, à renforcer l'opposition à R. Denktaş et à mobiliser au-delà des militants politiques

\footnotetext{
${ }^{9}$ Telle est notre conclusion, après la recherche menée à Chypre en 1999.

${ }_{11}^{10}$ Voir par exemple Le Monde du 13 août 1996.

11 Gilles Bertrand, "Les mobilisations des acteurs sociaux en faveur d'une réconciliation à Chypre face à l'impasse des négociations officielles", Cahiers de recherche du Groupe de recherches et d'études sur la Méditerranée et le MoyenOrient (GREMMO), n ${ }^{\circ}$, 2001, pp. 63-75.
} 
"habituels"12. Cette mobilisation s'est traduite, dès l'été 2000, par une contestation sans précédent du régime Denktaş et du rôle des militaires turcs dans le fonctionnement de la KKTC, dans le contexte d'une grave crise économique et financière. Plus de 10000 personnes $^{13}$ manifestent le 18 juillet 2000 à l'appel d'une plateforme de 41 organisations non gouvernementales baptisée «Ce pays est le nôtre » (Bu memleket bizimdir). Dans un premier temps, le régime pense étouffer le mouvement par la répression et les menaces "routinières"14 : cette fois, c'est le journal quotidien d'opposition Avrupa qui est visé : son directeur, Şener Levent, et plusieurs journalistes sont accusés d'espionnage au profit des Chypriotes grecs et emprisonnés (juillet 2000). L'imprimerie du journal est incendiée, les locaux de la rédaction détruits deux fois par des bombes (2000 et 2002). Depuis, toujours sous la menace de nombreux procès, Ş. Levent a rebaptisé le journal Afrika, car selon lui, la KKTC est davantage une république bananière africaine qu'un État de droit européen.

Une autre plateforme, plus modérée, "Une Vision commune de la société civile chypriote turque ", est présentée à la presse le 9 août 2002. Elle regroupe 86 puis 92 organisations non gouvernementales soit plus de 38000 personnes membres de ces associations. L'un de ses instigateurs est Ali Erel, président depuis deux ans de la chambre de commerce chypriote turque. Or Ali Erel appartient à un centre-droit plutôt modéré, très sceptique vis-à-vis d'une possible réunification de Chypre $^{15}$, du moins jusqu'aux graves événements de l'année 2000 et à la détérioration de la situation économique et politique de la zone nord. Mais la perspective de l'adhésion à l'UE a eu un effet décisif, point que nous allons aborder ensuite.

12 Nous sommes ici face à une problématique devenue classique en Europe de l'Ouest et notamment en France : celle des nouvelles formes de militantisme (ou de la nouvelle militance).

${ }^{13}$ La zone nord compte 200000 habitants.

${ }^{14}$ Les menaces de mort sont fréquentes contre une poignée d'opposants irréductibles, tel Alpay Durduran, dirigeant de plusieurs partis d'opposition depuis 1976; elles vont plus rarement jusqu'à l'attentat à la bombe (du moins entre 1974 et 2000), même si deux voitures d'Alpay Durduran ont sauté (nuitamment) dans les années 1990; le meurtre, celui du journaliste Kutlu Adalı (1996), reste exceptionnel.

${ }^{15}$ Voir le livre de l'un de ses très proches amis, l'ancien président de l'association des hommes d'affaires chypriotes turcs, Mustafa Damdelen, Scenarios to the Cyprus Problem, European Union and Sustainable Peace, Nicosie, publié à compte d'auteur, 1998. Lors de notre entretien à Nicosie-nord en mai 1999, Mustafa Damdelen se montrait très sceptique quant au fonctionnement d'une fédération à Chypre. Il milite désormais, avec Ali Erel, au sein de la Chambre de commerce, pour le plan Annan qui propose précisément une fédération! 


\section{L'impact de la classe politique et l'opinion publique turques}

L'évolution de la situation dans l'île jusqu'à la fin des années 1990 ne provoque guère de remise en cause du consensus sur la question chypriote en Turquie. C'est véritablement avec la crise de 2000 que l'opinion publique turque découvre que les Chypriotes turcs ne vivent pas dans le paradis qu'ont créé pour eux l'armée turque et $\mathrm{R}$. Denktaş. Les images des manifestations, diffusées par les chaînes de télévision turques, créent un malaise et rappellent notamment le mouvement citoyen des années 1996-1998 baptisé " une minute d'obscurité pour la lumière » (sous-entendu : sur les affaires de corruption) en Turquie. Les méthodes employées contre les opposants politiques et surtout contre les journalistes d'Avrupa/Afrika font écho à celles de la "sale guerre" contre les séparatistes kurdes et contre les journalistes turcs qui n'approuvaient pas la politique officielle. La différence, bien entendu, est que le mouvement chypriote turc est pacifique : il n'est donc pas possible de présenter l'argument de la lutte anti-terroriste. Plus grave pour les nationalistes turcs : les Chypriotes turcs refusent déjà, implicitement, l'union, même à long terme, à la mère-patrie (Anavatan) turque. Ils réclament au moins l'émancipation de leur enfant-patrie (Yavruvatan). D'où la crispation d'une partie de l'élite politique turque et le soutien accordé par le Premier ministre Bülent Ecevit à $\mathrm{R}$. Denktaş en 2000, y compris dans la répression. Offense suprême, les Chypriotes turcs manifestent, à partir de novembre 2002, en faveur du plan Annan et donc de la réunification de l'île! Le nouveau gouvernement AKP pouvait donc prévoir le «oui » au plan Annan lors du référendum.

Il faudrait discuter, ici, de la popularité réelle de R. Denktaş en Turquie. Les avis à ce sujet sont très partagés. Certains observateurs affirment que le président de la KKTC est considéré comme un héros du nationalisme turc et que sa crédibilité n'a été entamée qu'après la crise de 2000. Peu ménagé par les médias turcs depuis lors, R. Denktaş (né en 1924) apparaît désormais comme un dirigeant d'une autre génération, à l'instar de Bülent Ecevit ou de Süleyman Demirel; il devrait donc prendre lui aussi sa retraite, semblent dire certains médias turcs. Pour d'autres observateurs, R. Denktaş n'était guère connu du grand public avant la crise de 2000; c'est donc essentiellement grâce à ses réseaux dans la classe politique et l'armée turques qu'il était en position de force à Chypre-nord. En l'absence d'enquête sérieuse auprès de l'opinion publique turque, nous en sommes réduits à considérer les deux hypothèses comme partiellement valables : R. Denktaş a toujours été célébré dans les milieux nationalistes, et notamment au Parti du mouvement national 
$\left(\mathrm{MHP}^{16}\right)$ qui est un parti de militants aux idées largement diffusées. S'il est indéniablement connu du grand public aujourd'hui (notamment par de nombreux passages à la télévision depuis 2000), son image n'est guère positive et a aidé l'actuel gouvernement à le marginaliser ${ }^{17}$, afin de faire passer le plan Annan et de prouver ainsi à l'Union européenne que la Turquie prenait très au sérieux sa candidature et était prête à de douloureux sacrifices pour elle.

\section{Les candidatures à l'Union européenne}

Les deux candidatures sont liées depuis le compromis du 6 mars 1995 préparé par le ministre français des Affaires étrangères de l'époque, Alain Juppé (présidence française semestrielle de l'UE). Le gouvernement turc obtient alors la signature d'un accord d'union douanière avec l'UE pour lequel le gouvernement grec lève son veto. En échange, l'ouverture des négociations d'adhésion de la république de Chypre est finalement ${ }^{18}$ mise sur agenda : six mois après la fin de la Conférence intergouvernementale de 1996 (qui aboutit au traité d'Amsterdam). Tansu Çiller, Premier ministre à l'époque, eut beau jeu d'affirmer que le compromis était interne à l'UE et ne l'engageait en rien. Elle était bien sûr informée de ce compromis et l'a tacitement accepté. En effet, sachant pertinemment que la menace du veto grec existait, elle n'a fait aucune autre proposition (sur le règlement du différend en mer Égée, par exemple) pour parvenir à sa levée. Dans cette

\footnotetext{
${ }^{16}$ Milliyetçi Hareket Partisi.

${ }^{17}$ R. T. Erdoğan déclare le 2 janvier 2003 (il n'est alors pas encore Premier ministre mais dirigeant de l'AKP déjà au pouvoir) que « Chypre n'est pas l'affaire personnelle de M. Denktaş " (traduction dans Le Monde du 5-6 janvier 2003 et cette citation a été reprise par toute la presse internationale, par exemple The Guardian du 3 janvier 2003), ce qui constitue en soi un virage à 180 degrés de la politique chypriote de la Turquie. Lors de nos entretiens au ministère turc des Affaires étrangères en 1999, la réponse invariable (sinon obligée) était : «il n'y a aucune différence entre la politique de Rauf Denktaş et celle de la Turquie " sur Chypre, comme si le premier était le ministre turc des Affaires chypriotes en quelque sorte.

${ }^{18}$ La candidature de la république de Chypre à l'UE date de 1988, mais elle a été ajournée par le président Vassiliou (1988-1993) en raison de l'opposition d'AKEL qui était son principal soutien au parlement. Comme mentionné plus haut, la candidature fut relancée par Glafcos Clerides en 1993. Mais, malgré un Avis favorable de la Commission européenne, les États-membres restèrent dans l'expectative en raison de la division de l'île et des difficultés prévisibles avec la Turquie dont la candidature venait d'être rejetée et par la Commission et par le Conseil européen (décembre 1989).
} 
affaire, comme dans d'autres concernant les relations UE-Turquic, les dirigeants politiques turcs ont davantage accepté des décisions (decisiontakers) que fait des propositions (decision-makers).

Leur attitude dans les années suivantes, notamment à partir de 1998 et de l'ouverture effective des négociations UE-république de Chypre, a été empreinte d'une indéniable naïveté, à moins qu'il ne s'agisse d'une méconnaissance des mécanismes de décision au sein de l'UE.

Les dirigeants turcs des années 1990 ne pouvaient espérer, en effet, que les négociations d'adhésion de la république de Chypre échouent ou traînent en longueur. Car, une fois la décision d'ouvrir des négociations d'adhésion prise par le Conseil européen, le processus ne peut être retardé voire bloqué que si l'État candidat ne respecte pas le calendrier fixé et/ou ne répond pas aux demandes de la Commission en ce qui concerne la transposition de l'acquis communautaire dans sa législation nationale. Or la candidature de la république de Chypre apparaissait dès le départ comme très difficile sur le plan politique mais pas d'un point de vue économique et technique. Certes, il $\mathrm{y}$ avait le problème des sociétés off-shore. Une réforme de la législation et une politique moins laxiste de la part des autorités financières de la république de Chypre ont rapidement réglé la question, au moins à la satisfaction de Bruxelles. Par ailleurs, la république de Chypre est l'un des premiers États européens à avoir respecté les fameux «critères (macroéconomiques) de Maastricht » et le premier parmi les États candidats. Il était donc clair que ce n'était pas ces critères qui pouvaient freiner le processus d'adhésion; et, de fait, Chypre est le premier candidat à avoir clos tous les chapitres de la négociation.

C'est donc plutôt sur le terrain politique que les dirigeants turcs et chypriotes turcs pouvaient tenter d'empêcher cette adhésion. R. Denktaş a constamment mis en cause la légitimité d'une candidature unilatérale chypriote grecque arguant du fait que lui, dirigeant des Chypriotes turcs légitimé par l'ONU, était contre. Son argument, suivant lequel la constitution de 1960 interdit à la république de Chypre de conclure des alliances dont ne feraient pas partie les trois puissances garantes, est faible. Il est tout de même curieux que le président de la KKTC, dotée d'une constitution (adoptée par référendum en 1985) et qui prétend à la reconnaissance internationale en tant qu'État, invoque une constitution qu'il considère officiellement comme caduque depuis $1963 \ldots{ }^{19}$ En fonction de cet

${ }^{19}$ Sur les positions officielles turque et chypriote turque vis-à-vis de la constitution chypriote de 1960, voir notamment Necati Ertekün, The Cyprus Dispute and the Birth of the Turkish Republic of Northern Cyprus, Nicosie, K. Rustem \& Bros, $2^{\text {ème }}$ édition, 1984; Zaim Necatigil, The Cyprus Question and the Turkish Position in 
argument, il a refusé d'envoyer une délégation chypriote turque aux négociations UE-Chypre, ce à quoi l'invitaient l'UE et Glafcos Clerides. Il s'agit peut-être d'une grave erreur de sa part. Il a en effet perdu bien des occasions de bloquer le processus de l'intérieur, en multipliant, par exemple, les demandes financières et de dérogations, tout en laissant croire ${ }^{20}$ qu'il était favorable à l'adhésion - ce qui aurait pu être positif, indirectement, pour la candidature turque.

Les gouvernements turcs successifs de T. Çiller à B. Ecevit ont conduit une politique chypriote qui n'a en rien favorisé la candidature turque mais plutôt souligné que la Turquie était l'obstacle à un règlement de la question chypriote. T. Çiller (jusqu'en janvier 1996), puis Necmettin Erbakan (199697), Mesut Yılmaz (1997-98) et Bülent Ecevit (1999-2002) ont tous brandi la menace d'une «intégration» de la KKTC à la Turquie en cas $\mathrm{d}^{\prime}$ «intégration» de la république de Chypre ${ }^{21}$.

A sa décharge, $T$. Çiller, la première, est prise au dépourvu par le Président Süleyman Demirel qui signe, le 28 décembre 1995, une " déclaration commune » avec R. Denktaş sur la question de Chypre et le développement des relations Turquie-KKTC ${ }^{22}$. Les deux présidents indiquent que l'union douanière UE-Turquie (que vient de conclure T. Çiller) ne peut «entraver» les relations commerciales et économiques Turquie-KKTC (point 7). Ceci est faux : par l'union douanière, la Turquie s'engage, en principe, à s'aligner sur la politique commerciale de l'UE, y compris lorsque cette politique implique un embargo contre la KKTC... Un an plus tard, les deux présidents signent une deuxième déclaration commune ( 20 janvier 1997), laquelle porte principalement sur la disparition des barrières douanières entre la Turquie et la KKTC. Cette fois, le gouvernement de coalition dirigé par N. Erbakan - et dont $\mathrm{T}$. Çiller est ministre des Affaires étrangères - est partie prenante et

International Law, Oxford, Oxford University Press, 1989; la revue Perceptions (Ankara), "Special Issue on Cyprus", Vol. 4, n³, Septembre-Octobre 1999; et Two Peoples and Two States in Cyprus at the Eleven Hour?, Proceedings of a seminar held at the Turkish Embassy in London, 16 février 2001.

${ }^{20}$ Après tout, l'un des proches de R. Denktaş nous a confié, lors d'un entretien en juin 1999, que «nous faisons semblant de négocier à l'ONU », alors pourquoi pas auprès de l'UE?

${ }^{21}$ Officiellement appelée en Turquie l'Administration grecque de Chypre Sud (Güney Kıbrıs Rum Yönetimi).

${ }^{22} \mathrm{La}$ version anglaise de cette Déclaration est publiée par Briefing (Ankara), $\mathrm{n}^{\circ} 1073,1^{\text {er }}$ janvier 1996, p. 8. 
approuve $\mathrm{e}^{23}$. Une troisième déclaration, co-signée par le vice-premicr ministre B. Ecevit cette fois, crée un Conseil d'association Turquie-KKTC (21 juillet 1997 ) et réaffirme le principe d'«intégration économique et financière». Dans un tel contexte, le Premier ministre M. Yilmaz peut difficilement s'attendre à ce que le Conseil européen réuni à Luxembourg (12-13 décembre 1997) prenne une décision favorable à la Turquie. Mais la politique chypriote de la Turquie n'est évidemment pas le seul paramètre pris en compte par les chefs d'État et de gouvernement de l'UE.

La décision du sommet d'Helsinki, deux ans plus tard (10-11 décembre 1999) doit bien sûr beaucoup au rapprochement gréco-turc. Mais la position turque vis-à-vis de Chypre ne s'est pas infléchie. Alors que les ministres des Affaires étrangères İsmail Cem et Yorgos Papandréou discutent du règlement des différends bilatéraux, le Premier ministre B. Ecevit garde la haute main sur le dossier chypriote, confié au ministre d'État Şükrü Sina Gürel, un "faucon"24. B. Ecevit, célébré comme un héros par le régime Denktaş, cultive en Turquie son image de patriote qui a décidé de l'intervention militaire en 1974. Il n'est pas, par ailleurs, le plus ardent partisan de la candidature turque à l'UE; c'est le vice-Premier ministre $\mathbf{M}$. Yılmaz qui est chargé de ce dossier. Quant à l'autre vice-Premier ministre, Devlet Bahçeli, il dirige le MHP, le plus fidèle soutien de R. Denktaş. Ces contradictions rendent la politique européenne de la Turquie illisible et aboutissent, finalement, à un sur-place : le statut de la Turquie n'évolue plus (de candidat potentiel à candidat qui a une date pour l'ouverture des négociations). Les menaces d'« intégration» de la KKTC à la Turquie n'impressionnent guère dans la mesure où la livre turque y est la monnaie en circulation depuis 1975 et où le régime survit déjà grâce à l'aide financière d'Ankara. Sans parler de la présence de l'armée turque...

Ces incohérences et discours martiaux contraignent les gouvernements des États-membres de l'UE à lever l'ambiguïté : bien entendu, la Turquie n'a pas de droit de veto sur la candidature chypriote. Le gouvernement turc a ainsi gâché l'opportunité de diviser le Conseil européen sur la question des pouvoirs des puissances garantes de Chypre et sur le risque d'élargir l'UE à un État partiellement occupé par un partenaire important... Les déclarations du ministre italien des Affaires étrangères du 26 août 1997, restent un

${ }^{23}$ Turkish Probe (n²13, 24 janvier 1997) indique même que T. Çiller déclare même que la Turquie «n'acceptera jamais les faits accomplis dans l'île ». Turkish Probe, $\mathrm{n}^{\circ} 213,24$ janvier 1997

${ }^{24}$ Voir son livre sur les relations gréco-turques Türk-Yunan Ilişkileri (1821-1993), Ankara, Ümit, 1993. Il fut ministre des Affaires étrangères après la démission d'İ. Cem (juillet 2002) jusqu'aux élections de novembre. 
phénomène isolé : après avoir parlé de l'existence de «deux républiques, deux gouvernements») à Chypre, Lamberto Dini estime que l'entrée de la république de Chypre dans l'UE ne peut se faire qu'avec l'accord de l'autre gouvernement ${ }^{25}$. Ce qui signifie la reconnaissance de la souveraineté de la KKTC et ouvre la voie à l'entrée simultanée de la Turquie et de Chypre dans I'UE. Le gouvernement italien démentira; et les gouvernements qui sont réservés vis-à-vis de la candidature de Chypre (comme celui de la France) se taisent. Il faut dire que le veto d'un État non membre sur la candidature d'un autre créerait un dangereux précédent : imagine-t-on la Russie menacer de veto la candidature de la Pologne et des Républiques Baltes à cause de l'enclave de Kaliningrad?

La cohérence l'emporte-t-elle avec le gouvernement monopartisan de Recep Tayyip Erdoğan? Au moins faut-il reconnaître que ce dernier a d'emblée $e^{26}$ pris des positions courageuses : par exemple en reconnaissant formellement le lien entre la candidature chypriote et celle de la Turquie que niaient ses prédécesseurs. Le gouvernement AKP a également le mérite de ne pas se draper dans sa dignité à chaque fois que l'UE invoque le règlement de la question chypriote comme l'une des conditions à l'adhésion de la Turquie. Il était tout de même étonnant, ces dernières années, d'entendre des responsables politiques turcs prétendre qu'il s'agissait d'une nouvelle exigence et qu'il n'en avait jamais été question lors du sommet d'Helsinki en 1999. Comme si l'UE pouvait, en principe, accepter en son sein un État occupant un autre État-membre! Cependant, force est de constater que certains dirigeants d'États-membres de l'UE n'ont pas été suffisamment clairs sur cette question, permettant ainsi à des décideurs turcs de s'engouffrer dans la brèche. Le silence des dirigeants européens sur Chypre, à l'exception notable mais aisée à comprendre des Britanniques, s'explique par leur volonté de ménager un allié et partenaire important, mais aussi par le fait que l'ONU suit le dossier. Les dirigeants de l'UE ont donc parié sur le scénario suivant : le processus d'adhésion de la république de Chypre allait créer une pression suffisamment forte sur la Turquie et $\mathrm{R}$. Denktaş pour les contraindre à négocier sous l'égide de l'ONU. Et l'UE n'aurait alors eu plus qu'à se féliciter d'une solution de la question chypriote dont elle aurait partagé le bénéfice politique avec l'ONU. L'inaction diplomatique de l'UE sur Chypre a été telle qu'il a fallu la victoire électorale de l'AKP et les déclarations encourageantes de ses dirigeants pour convaincre l'ONU de soumettre son plan de règlement de la question chypriote aux négociateurs.

${ }^{25}$ Turkish Probe, n²42, 29 août 1997 et Athens News, 28 août 1997.

${ }^{26}$ Lors de sa tournée européenne, et alors qu'il n'cst pas cncorc Prcmicr ministrc, cn octobre 2002. 
Cependant, les dirigeants politiques de l'UE n'ont jamais envisagé que les Chypriotes grecs puissent bloquer la réunification; les résultats des référendums du 24 avril 2004 étaient donc une surprise. L'UE se trouve désormais dans une situation délicate vis-à-vis de la Turquie : celle-ci occupe toujours une partie de la république de Chypre mais presque à son corps défendant désormais! Utiliser la question chypriote pour lui refuser l'adhésion devient donc extrêmement difficile.

\section{Le plan Annan}

Le plan Annan a été présenté le 11 novembre 2002, soit moins de dix jours après la victoire de l'AKP! Initialement, le Secrétaire général a demandé que les dirigeants chypriotes acceptent ou rejettent ce plan avant le sommet européen de Copenhague (11-12 décembre). Mais il a lui-même accepté une révision (10 décembre) et une nouvelle date limite (28 février 2003). La troisième version du plan (26 février) a été rejetée par $\mathrm{R}$. Denktaş - qui a refusé de la soumettre à référendum - après un "sommet de la dernière chance" à La Haye le 10 mars 2003. Après cette date, les désaccords entre le gouvernement turc et le président de la KKTC ont été de plus en plus saillants. L'arrivée au pouvoir de M.A. Talât à Nicosie-Nord a conforté la stratégie de marginalisation de $\mathrm{R}$. Denktaş. L'un des rares dirigeants de la gauche chypriote turque à n'avoir jamais critiqué ouvertement le rôle de la Turquie à Chypre, M.A. Talât rendait possible la relance des négociations. La Conseil national de sécurité turc $\left(\mathrm{MGK}^{27}\right)$ approuvait cette relance lors de sa réunion mensuelle du 23 janvier 2004. L'ultime cycle de négociations sur le plan Annan (version 4) s'est alors tenu à Nicosie (19 février-22 mars) puis à Bürgenstock, en Suisse, du 24 au 31 mars 2004. Malgré l'absence d'un accord Denktaş-Papadopoulos, Kofi Annan a soumis une cinquième version de son plan aux corps électoraux des zones nord et sud de Chypre ( 24 avril). $76 \%$ des Chypriotes grecs ont voté contre tandis que $65 \%$ des électeurs de la KKTC votaient pour.

Le plan Annan, comme les plans onusiens précédents et les résolutions du Conseil de sécurité, recommande le principe d'une «fédération bizonale bicommunautaire $»^{28}$. Dans le plan Annan, «Chypre a une seule personnalité légale internationale et une seule souveraineté " (article 2 de l'accord de

\footnotetext{
${ }^{27}$ Milli Güvenlik Kurulu.

${ }^{28}$ L'ONU soutient les négociations entre le dirigeant de la communauté chypriote grecque qui est le Président de la République de Chypre et le dirigeant de la communauté chypriote turque qui est, de fait, le Président élu de la KKTC, ce qui constitue déjà une forme de reconnaissance.
} 
Fondation proposé) et il y a « une seule citoyenneté ». Mais c'est une fédération qui compte un «État commun», baptisée (dès la version 3) «République unie de Chypre » (United Cyprus Republic) et deux «États constituants ». La véritable innovation est la référence au modèle suisse, et non plus belge qui était la base de travail depuis $1997^{29}$. Ainsi, l'accord de Fondation énonce, en son article 2, que « le statut et les relations de l'État de Chypre, le gouvernement de l'État commun et ses États constituants, a pour modèle le statut et les relations de la Suisse, son gouvernement fédéral et ses cantons ».

Sans entrer davantage dans les détails du système constitutionnel proposé, disons que ce plan représente un bon compromis entre les revendications chypriotes grecques (une réintégration la plus poussée possible de manière à revenir quasiment à la constitution de 1960) et la position officielle chypriote turque, à savoir une confédération - en réalité une association librement consentie entre deux États souverains, préalablement reconnus internationalement. Cette dernière solution est tout simplement inacceptable pour les Nations unies, tant pour le Secrétariat général que pour le Conseil de sécurité et l'Assemblée générale, dans la mesure où elle signifierait la reconnaissance du fait accompli, la partition de l'île par la force. Cependant, les États constituants sont dotés d'une autonomie suffisamment large pour satisfaire sinon les radicaux, du moins les nationalistes chypriotes turcs modérés... Et toute la gauche (qui représente une - petite - moitié de l'électorat de la KKTC). La création d'un État constituant chypriote turc peut permettre à Ankara de sauver la face : le fait accompli de 1974 ne serait pas reconnu, mais les Nations unies reconnaîtraient en partie la validité du concept de partage de l'île (taksim) et d'institutions séparées pour les Chypriotes turcs, ce à quoi avaient œuvré les constitutionnalistes turcs participant à la rédaction de la constitution de 1960. D'autre part, au sein du Conseil présidentiel proposé, qui serait de six membres ${ }^{30}$, les portefeuilles des Affaires étrangères et des Affaires européennes ne pourraient être confiés à deux personnes issues du même État constituant : l'un des deux serait donc un Chypriote turc ${ }^{31}$. Or on peut supposer qu'il n'agirait pas à

${ }^{29}$ C'est ce modèle qu'étudient Michael Emerson et Nathalie Tocci dans Cyprus as Lighthouse of the East Mediterranean. Shaping Re-unification and EU Accession Together, Bruxelles, Centre for European Policy Studies, 2002.

30 Dotés du droit de vote. Selon la version 5 du plan, il y aurait aussi un nombre encore indéterminé de membres sans droit de vote.

31 Dans la version 5 du plan, il est prévu que les premiers titulaires seraient un Chypriote turc aux Affaires étrangères et un Chypriote grec aux Affaires européennes. 
l'encontre de l'intérêt national de la Turquie. Et la même chose est vraisemblable pour son collègue chypriote grec chargé de l'autre portefeuille, dans la mesure où la politique extérieure suivie serait définie par le Conseil... Les diplomates turcs n'auraient donc plus un adversaire en face d'eux, comme cela se produit actuellement dans les enceintes internationales quand arrive le représentant de la république de Chypre, mais un allié... Sans compter sur le délégué de la République hellénique...

Un autre aspect du plan le rend tout à fait acceptable par la Turquie. Il s'agit de l'ajustement territorial. Les deux États constituants couvrent les actuelles zones nord et sud. Mais un ajustement territorial est prévu de manière à rééquilibrer la situation dans la mesure où les Chypriotes turcs ( $18 \%$ de la population en 1974) vivent sur $37 \%$ de la superficie de l'île. Le plan Annan ne leur en laisserait que 28,6\% (version 3$)^{32}$. Ceci n'est pas une innovation majeure par rapport au plan précédent, «l'Ensemble d'Idées» présenté par Boutros Boutros-Ghali en 1992 (29\%). Hormis dans les zones concernées par cet ajustement territorial, le droit au retour des réfugiés est restreint par le plan Annan. Il y a un système de compensation pour les propriétés des réfugiés qui ne pourront ou ne voudront pas retourner chez eux. Mais ils ne seront pas obligés de vendre. Ils pourront mettre leurs propriétés en location. La population chypriote turque resterait donc regroupée et donc plus facile à défendre en cas de nouveaux troubles intercommunautaires, hypothèse à laquelle nous ne croyons d'ailleurs pas, dans la mesure où Chypre sera membre de l'UE.

En ce qui concerne la situation militaire, le plan Annan ne remet pas en cause les traités de 1960, l'existence de puissances garantes (GrandeBretagne, Grèce et Turquie), et la présence de troupes grecques et turques dans l'île. Cette présence est limitée à 6000 militaires (version 3), puis à 3000 après 2011 (version 5) et finalement à 950 Grecs et 650 Turcs après 2018. Il s'agit d'une concession majeure de l'armée turque (35000 soldats actuellement), puisqu'il s'agirait à terme d'un retour au traité de garantie de 1960. Or, jusqu'alors, un retrait militaire d'une telle ampleur n'avait jamais été envisagé par l'armée turque.

Le maintien de l'existence de puissances garantes prévu par le plan Annan permettrait de sauvegarder l'intérêt national turc à Chypre, si cet intérêt national comprend la présence de troupes dans l'île. Reste que le contenu de cet intérêt national turc à Chypre n'est pas forcément clair : s'agit-il de

${ }^{32}$ En cas d'accord, le gouvernement britannique est prêt à céder la moitié de la superficie occupée par ses bases militaires aux Chypriotes. Ces bases représentent 99 miles $^{2}$ soit $1,2 \%$ de l'île. 46 miles $^{2}$ seraient cédés dont $90 \%$ à l'État constituant chypriote grec. 
maintenir un équilibre de la puissance en Méditerranée ou bien de protéger la communauté chypriote turque? Le débat reste ouvert ${ }^{33}$.

\section{L'évolution de la position turque à l'égard de Chypre}

Du strict point de vue de la politique étrangère - si celle-ci peut vraiment être isolée du contexte politique interne, ce qui est douteux ${ }^{34}-$ on peut dire que Chypre est devenu un dossier prioritaire à Ankara dès lors que les Britanniques envisageaient leur retrait et qu'ils invitaient le gouvernement turc à s'impliquer davantage dans les affaires de l'île sous couvert de la protection de la minorité chypriote turque, en 1954-55. Pourtant, les nationalistes turcs chypriotes avaient été éconduits dans un premier temps, au tout début des années 1950, lorsqu'ils s'étaient adressés à Ankara ${ }^{35}$. Ce fait est aujourd'hui largement occulté. Au contraire, officiels et grands médias nationaux soutiennent, ou soutenaient jusqu'à la fin des années 1990 , que le gouvernement turc avait toujours suivi de près les affaires chypriotes en raison de la position géographique de l'île et de la présence d'une minorité turque.

Ici apparaît l'argument géostratégique selon lequel la Turquie ne pourrait se permettre de voir Chypre contrôlée par une puissance hostile qui menacerait alors son flanc $\operatorname{sud}^{36}$. Un coup d'œil sur la carte semble indiquer que ce principe relève du bon sens : Chypre occupe en effet une position centrale en Méditerranée orientale et pourrait constituer une menace pour les principaux ports turcs méridionaux (Antalya, Mersin, Iskenderun). Elle pourrait également servir de porte-avions insubmersible pour attaquer la Turquie. Cependant, lorsque cette théorie est avancée pour la première fois, dans les années 1950, il paraît alors invraisemblable que l'URSS puisse prendre pied sur un territoire comprenant déjà des bases militaires

33 Sur la notion controversée d'intérêt national voir Dario Battistella, "L'intérêt national. Une notion, trois discours", in Frédéric Charillon (dir), Politique étrangère. Nouveaux regards, Paris, Presses de Science Po, 2002, pp. 139-166.

${ }^{34}$ Sur le débat théorique, voir Frédéric Charillon (dir), op. cit.

35 Niyazi Kizllyürek, Chypre : l'impasse des nationalismes, Athènes, Mavri Lista, 1999 , p. 65 et suivantes (en grec).

${ }^{36}$ Voir Erol Manisalı (universitaire et président de la fondation chypriote - Klbrls Vakfi - proche de Rauf Denktaş), Cyprus, Yesterday and Today, Istanbul, Der, 2000, pp. 127-132 et Mehmet Ali Birand, Thirty Hot Days, Nicosie, K. Rustem \& Bros, 1985, qui explique, au terme de son enquête sur l'intervention de 1974 que : «The reason for intervention : Greece must not be allowed to dominate this area of the Mediterranean » (p. 3). 
britanniques (pérennisées car maintenues sous souveraineté après l'indépendance en 1960). L'escadre soviétique de la Méditerranée n'est opérationnelle que dans les années 1970-80. Et les bonnes relations turcosoviétiques des années 1960 et 1970 démontrent à Ankara que Moscou a d'autres soucis et d'autres priorités au Moyen-Orient ${ }^{37}$. L'alliance soviétosyrienne même est une question d'opportunité pour Moscou et dirigée avant tout contre Israël. L'exemple de $\mathrm{Cuba}^{-}$- où s'installent des militaires soviétiques en 1962 malgré la base américaine de Guantanamo - n'est guère probant : Cuba est une île beaucoup plus grande que Chypre (où deux armées peuvent difficilement cohabiter sans se faire face) et les communistes chypriotes n'étaient notoirement ni disposés ni en mesure de se lancer dans une aventure révolutionnaire de type castriste.

La menace grecque est encore plus invraisemblable : à supposer que l'armée grecque ait jamais été en mesure de prendre le contrôle de l'île et d'y stationner des forces importantes sans susciter une réaction immédiate d'autres États, on l'imagine encore plus mal préparer un débarquement sur la côte turque méridionale. La "Grande Catastrophe" a dissuadé même les plus nationalistes des militaires grecs de ce genre d'aventure. En 1974, les chefs de l'armée grecque ont clairement signifié à la junte qu'il n'était pas question d'une guerre avec la Turquie. L'argument des années 1990 selon lequel la Grèce et la Syrie pourraient s'allier contre la Turquie et se servir de Chypre pour fermer la Méditerranée orientale à l'armée de l'air et à la marine turques relève de la pure politique-fiction ${ }^{38}$. Ni l'armée grecque ni la syrienne ne seraient en mesure d'aligner assez de navires et d'avions de combat pour une telle opération qui mésestimerait gravement l'écrasante supériorité militaire turque, notamment sur terre. On ne voit pas trop non plus pourquoi et comment un État-membre de l'OTAN et de l'UE, la Grèce, s'allierait avec la Syrie. Le coût en serait, d'entrée de jeu, énorme. Et à supposer même qu'il

\footnotetext{
${ }^{37}$ Voir notamment Didier Billion, La Politique extérieure de la Turquie. Une longue quête d'identité, Paris, L'Harmattan, "Comprendre le Moyen-Orient », 1997, pp. 68-84 et pp. 214-222 et Semih Vaner, "La Turquie entre l'Occident-patron et le "'Grand voisin du Nord"”, in Zaki Laïdi (dir.), L'URSS vue du Tiers-Monde, Paris, Khartala, 1984, pp. 95-120.

38 Un accord militaire gréco-syrien qui daterait de 1995 est évoqué par plusieurs chercheurs, notamment Élie Kheir, "La Turquie et le Moyen-Orient", in Hasan B. Elmas, Regards sur les ambitions turques, Paris, Syllepse, 1999, p. 124 et Philip Robins, Suits and Uniforms. Turkish Foreign Policy since the Cold War, Londres, Hurst, 2003, pp. 171-172. Mais il n'existe que des sources turques à ce sujet. Encore les diplomates turcs auprès desquels j'avais réalisé des entretiens en juin 1999 au ministère, à Ankara, n'avaient pas confirmé la réalité d'un tel accord!
} 
se trouve un Premier ministre grec assez fou pour envisager un tel accord, comment imaginer que la VIe flotte américaine n'interviendrait pas? Pourtant cette hypothèse d'une alliance gréco-syrienne, incluant qui plus est le Parti des Travailleurs du Kurdistan (PKK), était sérieusement évoquée par des personnalités turques aussi éminentes que Şükrü Elekdag $\breve{g}^{39}$, ancien ambassadeur à Washington et concepteur de la Zone de coopération économique de la mer Noire en 1990.

D'autres éléments décrédibilisent cette thèse : la rupture de tout lien entre la Grèce (autorités et/ou groupes plus ou moins liés au pouvoir) et le PKK après la capture d'Abdullah Öcalan en 1999; le rapprochement gréco-turc. On peut encore s'interroger sur l'importance stratégique de Chypre pour la Turquie à l'ère de la suprématie aérienne et de la surveillance satellitaire. Les avions de chasse F-16 turcs ont-ils réellement besoin d'une base à Chypre, à $80 \mathrm{~km}$ de la Turquie pour dominer le ciel de la Méditerranée orientale ${ }^{40}$ ? Pourtant, le chef d'état-major de l'armée turque, le général Hilmi Özkök a réaffirmé le 8 janvier 2003 devant la presse sa préoccupation face à la menace d'une «force assiégeante » (les forces armées grecques et chypriotes grecques) contre le sud et le sud-est du pays : « une solution à Chypre qui ne garantirait pas les besoins de sécurité de la Turquie signifierait, plus ou moins, le début d'un processus d'enfermement de l'Anatolie $\gg{ }^{41}$.

L'argument de la protection des Chypriotes turcs est lui aussi répété sans cesse depuis les années 1950. Jouant sur l'ambiguïté entre défense des droits de l'homme et des minorités et solidarité ethno-nationale, il motive la mobilisation sincère de nombreux Turcs en faveur d'un État indépendant turc à Chypre. Cependant, la politique de l'État turc par ailleurs, et principalement en ce qui concerne la question kurde, laisse penser que la solidarité ethno-nationale passe tout de même avant la défense des droits des Chypriotes turcs en tant que groupe minoritaire. Chypre a toujours suscité l'intérêt des panturquistes, y compris à l'époque (avant les années 1950) où

${ }^{39}$ Şükrü Elekdağ, 2“ 1/2 War Strategy”, Perceptions, Vol. 1, n¹, mars-mai 1996, pp. 33-57.

${ }^{40}$ En revanche, les bases souveraines britanniques ont une importance stratégique indéniable pour la Royal Air Force et l'US Air Force, comme l'ont montré les guerres du Koweït et d'Irak ( $2^{\text {ème }}$ et $3^{\text {ème }}$ guerres du Golfe) depuis la fin de la Bipolarité.

${ }^{41}$ Cité dans une dépêche de l'Agence France-Presse reprise par Le Monde du 11 janvier 2003. 
ceux-ci étaient les seuls à s'en préoccuper ${ }^{42}$. Chypre focalise donc l'attention des tenants de l'idéologie nationaliste, des militaires, mais aussi d'autres groupes : du crime organisé qui se livrent au blanchiment d'argent dans les casinos et les banques off-shore de la $\mathrm{KKTC}^{43}$; des politiciens qui utilisent la question chypriote et le répertoire nationaliste pour asseoir une légitimité qu'ils leur manquent en raison de leurs échecs politiques et économiques en Turquie même. R. Denktaş a parfaitement su exploiter cette situation en se constituant de puissants réseaux en Turquie. Ces liens nourrissent ainsi la légitimité des uns et des autres : $R$. Denktaş se présente comme l'interlocuteur privilégié, voire exclusif, de l'élite turque au pouvoir; les politiciens turcs, quant à eux, font de la surenchère sur le mode de "Je suis celui qui défend le mieux l'intérêt national turc, y compris, sinon surtout, à Chypre". Mais, ce faisant, ils ignorent ostensiblement les partis de l'opposition chypriote turque, pourtant généralement proches d'un ou de partis politiques turcs ${ }^{44}$. Il est encore trop tôt pour dire si l'arrivée au pouvoir du CTP à Nicosie-Nord et le "oui » au plan Annan des Chypriotes turcs vont durablement changer cette donne.

A la fin des années 1990, il est apparu clairement qu'Ankara était davantage préoccupée par la survie du régime Denktaş que de la volonté politique des Chypriotes turcs. C'est ainsi que le deuxième tour de l'élection présidentielle de 2000 n'a jamais eu lieu : les autorités turques, craignant une défaite de R. Denktaş, sont ouvertement intervenues pour que son concurrent (et Premier ministre) Derviş Eroğlu se retire après le premier tour alors qu'il était en ballottage favorable. Cette priorité au maintien de R. Denktaș au pouvoir a été encore plus manifeste durant la crise de l'été 2000. Là encore, le gouvernement AKP a inauguré une nouvelle approche du problème : R.T. Erdoğan s'est ainsi publiquement interrogé sur la légitimité de R. Denktaş après les grandes manifestations de l'hiver 2002-03. Il a déclaré que «si 30000 personnes manifestent en même temps au nord de Chypre, cela veut

\footnotetext{
${ }^{42}$ Leur dirigeant historique, Alparslan Türkeş, était d'ailleurs né à Chypre avant d'immigrer en Turquie où il avait fait une carrière militaire puis politique à la tête du MHP.

${ }^{43}$ Elle compte onze casinos (soit un pour 10000 habitants) et 80 banques off-shore (avant la crise financière de 2000-2001) selon le quotidien turc Yeni Yüzyıl du 26 janvier 1998.

44 Ainsi les fondateurs du TKP (Toplumcu Kurtuluş Partisi, Parti populiste/communautaire de Libération) étaient-ils proches voire militants du CHP durant leurs études en Turquie à la fin des années 1960-début des années 1970. C"est désormais le CTP qui est proche du CHP.
} 
dire qu'il se passe quelque chose ${ }^{45}$. Cette lapalissade a sonné comme un avertissement, d'autant que, dans le même discours, le futur Premier ministre a aussi précisé qu'il n'était pas favorable à la poursuite de la politique menée sur Chypre depuis trente à quarante ans. Pourtant, les dirigeants de l'AKP ont pour référence traditionnelle Adnan Menderes (Premier ministre des années 1950 qui inaugura la politique chypriote de la Turquie) et N. Erbakan (vice-Premier ministre en 1974 et qui reprochait alors à B. Ecevit de ne pas ordonner l'occupation de l'île entière). Changement d'autant plus radical pour un Abdullah Gül, Premier ministre de décembre 2002 à février 2003 puis ministre des Affaires étrangères après avoir été, dans le gouvernement Erbakan de 1996-1997, ministre d'État chargé de Chypre. Or, à l'époque, comme nous l'avons indiqué plus haut, le gouvernement turc menait une politique d'intégration et $\mathrm{N}$. Erbakan s'était rendu plusieurs fois en visite officielle à Chypre.

A. Gül a affirmé, à l'issue d'une réunion avec la troïka de l'UE ${ }^{46}$ le 13 novembre 2003, que " chacun sait que nous soutenons les efforts de l'ONU » et qu'il espérait une solution pour Chypre avant le $1^{\text {er }}$ mai 2004. Les ultimes négociations sur le plan Annan ( $1^{\text {cr }}$ trimestre 2004) ont prouvé que le gouvernement turc était prêt à "sacrifier» Chypre sur l'autel processus d'adhésion (pour reprendre la rhétorique nationaliste turque), tout comme il a pris de conscience de l'impopularité croissante de R. Denktaş et de la volonté de la majorité des Chypriotes turcs d'en finir avec le statu quo actuel pour intégrer l'UE. Il s'agit peut-être aussi (sans doute?) de la redéfinition de l'intérêt national turc : priorité à l'intégration de la Turquie à l'économiemonde via son intégration au sein de l'UE, "normalisation" de sa politique étrangère (notamment en se conformant aux grands principes du droit international, à Chypre comme en mer Égée) et des relations avec tous ses voisins proches et plus distants.

La politique chypriote de l'AKP est un élément de preuve supplémentaire, s'il en était encore besoin, que le gouvernement actuel a pour principale référence Turgut Özal, sa politique d'ouverture et de rapprochement avec la Grèce et d'intégration au grand marché européen. Reste que, comme Özal, R.T. Erdoğan a dû faire face à l'opposition des chefs de l'armée et d'une

\footnotetext{
${ }^{45}$ Cité (donc traduit) par Le Monde du 5-6 janvier 2003.

46 Il s'agit du ministre des Affaires étrangères de l'État assurant la présidence semestrielle (l'Italien Franco Frattini), de son collègue de la présidence suivante (l'Irlandais Brian Cowen) et du haut représentant pour la Politique étrangère et de sécurité commune (Javier Solanas) mais aussi du commissaire chargé de l'élargissement Günter Verheugen. Cette réunion a eu lieu après la publication du rapport 2003 de la Commission sur la candidature turque.
} 
partie de la haute fonction publique. Mais il a triomphé, semble-t-il, là où le premier a échoué. Les temps ont changé, la haute fonction publique, notamment au ministère des Affaires étrangères, n'est plus exactement la même qu'il y a dix-quinze ans. Les partisans de l'intégration européenne $-\mathrm{y}$ compris politique - sont plus nombreux aujourd'hui. Les perspectives de rapprochement et de coopération étroite avec les républiques turcophones d'Asie centrale sont bien moins prometteuses qu'au début des années 1990. L'Europe offre bien plus d'opportunités économiques et politiques. Pourtant, paradoxalement, l'électorat de l'AKP est, semble-t-il, majoritairement contre l'intégration européenne...

Le gouvernement AKP mène donc indéniablement une politique chypriote nouvelle, en rupture avec celle conduite depuis trente à quarante ans. Ce ne sont officiellement les autorités de la KKTC qui ont pris la décision d'ouvrir la ligne verte le 23 avril 2003, mais elle leur a été imposée par Ankara. Par cette mesure, le gouvernement turc a voulu signifier à $R$. Denktaş qu'il n'était certainement pas le seul maître à bord et que l'heure de la négociation était venue. Certes, cette ouverture a été suivie d'autres, en sens opposé. L'affaire des passeports ${ }^{47}$, la tournée électorale de R. Denktaş en Anatolie en décembre 2003 ou encore l'ouverture d'un consulat de la KKTC à Izmir peuvent laisser penser que le gouvernement a "soufflé le chaud et le froid" dans ce dossier. Mais chacune de ces mesures était manifestement destinée à calmer un secteur résistant particulier : les autorités de la KKTC ulcérées que les Chypriotes turcs voyagent désormais avec un passeport de la république de Chypre; les militaires et groupes politiques qui soutiennent $\mathrm{R}$. Denktaş; une partie du cours diplomatique toujours fidèle à la définition traditionnelle de l'intérêt national et à la défense de la KKTC. Le fait que M.A. Talat ait pu devenir Premier ministre sans opposition d'Ankara, les négociations et le référendum sur le plan Annan ont confirmé le tournant de la politique chypriote de la Turquie.

Après le vote négatif des Chypriotes grecs et celui, positif, de l'électorat de la zone nord (Chypriotes turcs et ressortissants turcs citoyens de la KKTC), la situation reste complexe. Du point de vue du droit international, la Turquie reste une puissance occupante à Chypre; elle pourrait toutefois se conformer au plan Annan et ne laisser que 6000 soldats à Chypre. Dans ce cas, la position des Chypriotes grecs deviendrait encore moins tenable. Les Chypriotes turcs, individuellement, en tant que citoyens de la république de Chypre de 1960 (suivant le droit international), entrent, de toute façon, dans

${ }^{47}$ A partir de juillet 2003, les Chypriotes turcs en transit en Turquie ont découvert qu'ils ne pouvaient plus voyager sans un passeport turc (et non de la république de Chypre ou la carte d'identité de la KKTC). 
l'UE le $1^{\text {er }}$ mai 2004. L'UE propose une aide économique à la KKTC et envisage d'établir des relations commerciales. Il semble donc que la Commission européenne et le Conseil européen considèrent que le gouvernement turc a fait tout son possible pour régler la question chypriote. Celle-ci ne peut donc plus être un motif de refus d'ouvrir les négociations d'adhésion de la Turquie à l'UE. 